Conclusion CRL discordance is of poor predictive value for adverse perinatal outcomeafter 14 weeks of gestation in either MC or DC twin pregnancies.

\section{PP.11 ONE THIRD OF NON-ANOMALOUS TERM STILLBIRTHS ARE ASSOCIATED WITH ABNORMAL FETAL GROWTH}

doi:10.1136/archdischild-2013-303966.292

AA Moraitis, GC Smith. University of Cambridge, Cambridge, UK

Background Approximately one third of all stillbirths occur at term. These losses are potentially preventable by early delivery at 37 weeks. One means of screening to detect babies at risk of term stillbirth would be to use ultrasound to assess abnormalities of fetal growth. However, there is limited information on the importance of fetal growth in determining the risk of stillbirth at term.

Methods/Results We studied registries of Scottish maternity and stillbirth data and identified 668,887 eligible records of term births since 1992. Birth weight was classified on the basis of sex and gestational age specific percentiles. The relationship between birth weight percentile and the risk of stillbirth (all causes except congenital anomaly) was assessed referent to birth weight percentiles in the range $20-80^{\text {th }}$. The risk (odds ratio [95\% CI] P) of stillbirth was increased with birth weight between the $1^{\text {st }}-3^{\text {rd }}$ percentile $(\mathrm{OR}=8.2[\mathrm{CI}: 6.8-9.9] \mathrm{P}<0.001), 4^{\text {th }}-10^{\text {th }}$ percentile $(\mathrm{OR}=3.5$ $[C I: 2.8-4.0] \mathrm{P}<0.001)$ and the $11^{\text {th }}-20^{\text {th }}$ percentile $(\mathrm{OR}=1.8$ [CI:1.4-2.2] $\mathrm{P}<0.001)$. The risk of stillbirth was also increasing among the largest infants: $98^{\text {th }}-100^{\text {th }}$ percentile (OR $=2.3$ [CI:1.73.1] $\mathrm{P}<0.001)$. The attributable fraction for stillbirth associated with these birth weight categories was 33\% (95\% CI: 28 to 37\%). The associations were minimally affected by adjusting for maternal characteristics, including smoking.

Conclusion Approximately one third of all term stillbirths are associated with growth abnormality. These data indicate that population screening for growth abnormality could identify babies at increased risk of term stillbirth.

\section{PP.12 WEIGHT DISCORDANCE AND PERINATAL MORTALITY IN TWINS: THE STORK MULTIPLE PREGNANCY COHORT}

doi:10.1136/archdischild-2013-303966.293

F D'Antonio, A Khalil, T Dias, A Bhide, B Thilaganathan. Fetal Medicine Unit, Division of Developmental Sciences, ST. George's Univeristy of London, London, UK

Objectives The main aim of this study was to ascertain the performance of BW and ultrasound EFW discordance in the prediction of perinatal loss in twin pregnancies.

Methods A retrospective study of all twin pregnancy births from a large regional cohort of 9 hospitals over a ten year period. Intertwin BW and ultrasound EFW discordance were related to stillbirth and neonatal death of one or both twins obtained from a mandatory national register. Receiver operating characteristic (ROC), survival and logistic regression analyses were performed to evaluate the contribution of weight discordance in determining perinatal loss.

Results A total of 2,161 twin pregnancies were included in the analysis. A BW discordance of $\geq 25 \%$ was associated with the highest AUC for the prediction of stillbirth and neonatal death (BW AUC $=0.72 ; 95 \%$ CI: $0.65-0.80)$. The perinatal loss in twins with a BW discordance of $\geq 25 \%$ was significantly greater $(60.9$ per 1,000 fetuses) than that in twins with an EFW discordance $<25 \%$ (8.6 per $1,000$ fetuses) ( $p<0.0001)$; the predictive accuracy was similar using either BW or ultrasound EFW discordance (EFW AUC $=0.69$; $95 \%$ CI: $0.62-0.77, p=0.62)$. Logistic regression analysis demonstrated that BW discordance and gestational age, but not chorionic- ity or individual fetal size percentile, were independently associated with perinatal mortality.

Conclusions An EFW discordance of $\geq 25 \%$ represents the optimal cut-off for the prediction of stillbirth and neonatal mortality irrespective of chorionicity or individual fetal size. A policy of increased fetal surveillance commencing from 26 weeks' gestation and elective delivery by 38 weeks might be reasonable.

\section{PP.13 PREVIOUS CAESAREAN DELIVERY AND THE RISK OF UNEXPLAINED STILLBIRTH: ANALYSIS OF 128,585 SECOND PREGNANCIES IN SCOTLAND, 1999-2008}

doi:10.1136/archdischild-2013-303966.294

AA Moraitis, GC Smith. University of Cambridge, Cambridge, UK

Background A previous study (Lancet 2003; 362:1779-84) reported an increased risk of unexplained stillbirth in women with previous caesarean delivery among women having second births in Scotland, 1992-1998. Subsequent studies have yielded heterogeneous results but have employed data, analytic approaches and interpretation of variable quality.

Methods/Results We replicated our previous methods and analysed 128,585 eligible singleton second births between 1999 and 2008. There were 88 stillbirths among 23,688 women with a previous caesarean (2.33 per 10,000 women per week) and 288 stillbirths in 104,897 women who previously delivered vaginally (1.67 per $10,000$ women per week, $\mathrm{P}=0.002)$. When analysed by cause, women with a previous caesarean had an increased risk (hazard ratio [95\%CI] P) of unexplained stillbirth $(1.47$ [1.12-1.94] $\mathrm{P}=0.006)$ and the excess risk was apparent from 34 weeks onwards $(1.75$ [1.23-2.49] $\mathrm{P}=0.002)$. When the analysis was confined to 96,988 women with linked records from the first and second pregnancy (confirming exact mode of previous delivery) the association was stronger $(2.12$ [1.55-2.88] $\mathrm{P}<0.001)$. Adjustment for maternal characteristics and first pregnancy complications had a minimal effect (1.97 [1.43-2.72] P < 0.001). The association was similar weather the previous caesarean was performed prior to labour (2.1[1.24 $3.80] \mathrm{p}=0.007)$ or during labour $(2.50$ [1.53-4.08] $\mathrm{p}<0.001)$ and when the analysis was confined to previous term births $(2.35$ [1.50 3.53] $\mathrm{p}<0.001$ ).

Conclusion We confirm that previous finding that previous caesarean delivery is a risk factor for unexplained stillbirth. The association is independent of maternal characteristics, obstetric outcome or the indication for the caesarean delivery.

\section{PP.14 PROGESTERONE MODULATES CERVICAL ANTIMICROBIAL IMMUNITY}

doi:10.1136/archdischild-2013-303966.295

1,2 $\underline{\mathrm{CP}}$ James, ${ }^{2} \mathrm{~N}$ Klein, ${ }^{2} \mathrm{M}$ Bajaj-Elliott, 'D Peebles. 'UCL Institute for Women's Health, London, UK; ${ }^{2}$ UCL Institute of Child Health, London, UK

Introduction Human beta defensins (HBD1, 2.3) have antimicrobial and immunomodulatory properties and are components of the cervical mucus plug. Vaginal progesterone delays delivery in women with cervical shortening, but the mechanism(s) underlying this effect remain undetermined. This study describes the expression of HBDs by cervical epithelia in response to stimulation with classical infective and inflammatory agonists and progesterone.

Methods The human endo-cervical cell-line End1/E6E7 and ectocervical cell-line Ect1/E6E7 were stimulated with bacterial (Lipopolysaccharide, LPS; Peptidoglycan, PGN) and inflammatory (Interleukin 1 beta, IL-1 $\beta$; Interferon gamma, IFN $\gamma$ ) agonists and progesterone for up to 24 hours. HBD secretion was assessed by ELISA. 
Results End1/E6E7 HBD1 expression did not respond to stimulation. HBD1 secretion by Ect1/E6E7 cells more than doubled in response to IL-1 $\beta(p=0.0002)$ and IFN $\gamma(p=0.02)$, and was suppressed by progesterone $(p=0.02)$. End1/E6E7 HBD2 release almost doubled in response to LPS $(p=0.02)$ and progesterone $(p=0.01)$. HBD2 expression by Ect1/E6E7 cells doubled in response to LPS $(p=0.005)$ and halved after stimulation with progesterone $(p=0.006)$. End1/E6E7 HBD3 expression increased in response to LPS $(p=0.0009)$, and almost doubled after stimulation with PGN $(p=0.0003)$ and IFN $\gamma(p=0.027)$. End1/E6E7 HBD3 secretion almost tripled in response to incubation with progesterone $(p=0.003)$.

Conclusion Bacterial products elicited differential expression of HBDs in End1/E6E7 and Ect1/E6E7 cells. Progesterone mediated an increase in $\mathrm{HBD} 2$ and $\mathrm{HBD} 3$ secretion by endo-cervical cells but suppressed HBD1 and HBD2 secretion by ecto-cervical cells; this effect may augment cervical host defence, representing a novel mechanism by which progesterone may contribute to delaying the onset of preterm labour.

\section{PP.15 MORTALITY, CONGENITAL ANOMALY, \& MATERNAL RISK FACTORS ACROSS ETHNIC GROUPS IN BIRMINGHAM}

doi:10.1136/archdischild-2013-303966.296

${ }^{1} \mathrm{AM}$ Tonks, ' $\mathrm{A}$ Williamson, ${ }^{2} \mathrm{D}$ Willams, ${ }^{1} \mathrm{JO}$ Gardosi. 'Perinatal Institute, Birmingham, UK; 'West Midlands Regional Genetics Service, Birmingham, UK

Background Stillbirth and infant mortality rates in Birmingham remain consistently above those seen in the West Midlands and England \& Wales. Over half of the maternity population are from minority ethnic groups.

Methods Numerator data 2006-2010 (deaths/anomalies) were selected from the regional, population-based, anomaly and mortality registers, covering a birth cohort of 85,734. Denominator data for 2010, including ethnic group, consanguinity, and folate use, were available within the regional denominator database (www. pi.nhs.uk/peer/peerdata collection.htm).

Results Deaths from congenital anomaly comprise $29.3 \%$ of stillbirths and infant deaths in Birmingham. Mortality rates were significantly higher in Pakistani (odds ratio 3.0) and Bangladeshi mothers (odds ratio 2.1) compared to White Europeans. Pakistani mothers had significantly higher mortality rates from metabolic disorders, neural tube defects, and renal anomalies. In terms or primary and secondary screening, the prevalence of antenatal folate use was low in most minority ethnic groups. $49.4 \%$ of stillbirths and infant deaths had at least one anomaly that was amenable to detection by fetal anomaly screening programmes. However, in $5.1 \%$ of Birmingham births, the first booking appointment took place at 20 weeks or later. Pakistani mothers have the highest rates of consanguineous unions (49.9\%, CI 48.1-51.7, compared to $15.9 \%$ (CI 15.3-16) for all ethnic groups combined.

Conclusion Ethnic groups in Birmingham have an excess of perinatal mortality due to congenital anomalies. Interventions for these groups need to focus on improved folate uptake, timely access to screening services, and referral for genetic risk assessment.

\section{PP.16 PREGNANCY OUTCOMES IN WOMEN WITH KNOWN SICKLE CELL DISEASE: A CASE CONTROL STUDY FROM A DEDICATED TERTIARY JOINT OBSTETRIC SICKLE CELL CLINIC}

doi:10.1136/archdischild-2013-303966.297

'S Nanda, ${ }^{3} \mathrm{~S}$ Robinson, 'S Sankaran, 'P Kyle, ${ }^{2} \mathrm{~K}$ Nicolaides, 'E Oteng-ntim, A Weaver 'Women's Health, King's Health Partners, St Thomas' Hospital, London, UK; ' ${ }^{2 H a r r i s}$ Birthright Research Centre for Fetal Medicine, King's College Hospital, London, UK; ${ }^{3}$ Department of Haematology, Guy's and St Thomas' Hospital, London, UK
Introduction Sickle cell disease (SCD) in pregnancy has been associated with adverse pregnancy outcomes. However, with a multidisciplinary approach and surveillance, there is a suggestion of improvement in the overall outcome. The aim of this study was to evaluate the pregnancy outcomes in women with known SCD in a singleton pregnancy seen in our clinic, and to assess whether the presence of SCD alters the maternal serum analytes (Free B-human chorionic gonadotrophin and Pregnancy-associated plasma protein - A) at combined screening.

Methods Case-control study of all pregnant women with SCD undergoing combined screening from 01/01/2008 to 31/12/2011. Each case was matched with 3 non-SCD controls. Pregnancy outcomes in the two groups were compared.

\section{Abstract PP.16 Table}

\begin{tabular}{lll}
\hline Results & SCD $(\mathbf{n}=\mathbf{5 4})$ & Controls $(\mathbf{n}=\mathbf{1 6 5})$ \\
\hline Pre-eclampsia, n (\%) & $7(12.9)^{*}$ & $3(1.8)$ \\
Chronic hypertension, n (\%) & $1(1.8)$ & $4(2.4)$ \\
Live birth & $53(98.1)$ & $164(99.3)$ \\
CS, n (\%) & $20(37.7)$ & $42(25.6)$ \\
Vaginal, n (\%) & $33(62.3)^{*}$ & $122(74.4)$ \\
Gestation at delivery, wks, median (IQR) & $38(37-39)$ & $39(38-40)$ \\
Birth weight (grammes), median (IOR) & $3060^{*}$ & $\mathrm{AB3345}$ \\
B-HCG MoM, median (IOR) & $(2698-3280)$ & $(3000-3563)$ \\
PAPP-A MoM, median (IOR) & $1.2(0.65-1.85)^{*}$ & $1.05(0.62-1.63)$ \\
\hline
\end{tabular}

${ }^{*} \mathrm{P}<0.05$

Conclusion Women with SCD, when followed closely from the first trimester, have successful pregnancy outcomes. However, our data suggests an increased incidence of pre-eclampsia and lower birth weights compared to non-SCD controls. There was also a significant difference noted in the maternal serum analytes. Larger prospective studies are therefore required to assess the impact of SCD on combined screening.

\section{PP.17 PRE-GESTATIONAL DIABETES AND THE RISKS OF FETAL AND INFANT DEATH IN NORMALLY-FORMED OFFSPRING}

doi:10.1136/archdischild-2013-303966.298

'PWG Tennant, 'SV Glinianaia, ${ }^{2}$ RW Bilous, ${ }^{1,3} \mathrm{~J}$ Rankin, ${ }^{1,3} \mathrm{R}$ Bell. 'Institute of Health \& Society, Newcastle University, Newcastle-upon-Tyne, UK; ' James Cook University Hospital, South Tees NHS Trust, Middlesbrough, UK; ${ }^{3}$ Regional Maternity Survey Office, Newcastle-upon-Tyne, UK

Background Pre-gestational diabetes is associated with substantially increased risks of congenital anomalies, but the impact on normally-formed offspring is less well explored. This study explored the risks of fetal and infant death, examining the influence of $\mathrm{HbA1c}$, in normally-formed offspring of women with pregestational diabetes.

Methods All normally-formed singleton pregnancies in Northern England delivered during 1996-2008 were identified from the Northern Diabetes in Pregnancy Survey. Fetal ( $\geq 20$ weeks gestation) and infant deaths were identified from the Northern Perinatal Morbidity and Mortality Survey. Relative risks were estimated by comparing the prevalence rates between those with and without diabetes. The associations between peri-conception and third trimester $\mathrm{HbA1c}$ with each outcome were examined by logistic regression.

Results 400,158 normally-formed singletons were registered during the study period, including 1548 in women with pre-gestational diabetes. There were 46 fetal and 10 infant deaths following pregnancies in women with diabetes. 